\title{
Treatment of Alcoholic Liver Disease
}

\author{
Ina Bergheim ${ }^{\mathrm{a}}$ Craig J. McClain ${ }^{\mathrm{b}}$ Gavin E. Arteel $^{\mathrm{a}}$ \\ ${ }^{a}$ Department of Pharmacology and Toxicology and James Graham Brown Cancer Center, \\ University of Louisville Health Sciences Center, and ${ }^{\mathrm{b}}$ Department of Medicine, University of Louisville, and \\ Veterans Administration, Louisville, Ky., USA
}

\section{Key Words}

Alcoholic liver disease $\cdot$ Cirrhosis, alcoholic $\cdot$ Hepatitis . Nutrition intervention $\cdot$ Pentoxifylline

\begin{abstract}
Alcoholic liver disease (ALD) remains a major cause of morbidity and mortality worldwide. For example, the Veterans Administration Cooperative Studies reported that patients with cirrhosis and superimposed alcoholic hepatitis had a 4-year mortality of $>60 \%$ (worse than many common cancers such as breast and prostate). The cornerstone for therapy for ALD is lifestyle modification, including drinking cessation and treatment of decompensation, if appropriate. Nutrition intervention has been shown to play a positive role on both an in-patient and out-patient basis. Corticosteroids are effective in selected patients with alcoholic hepatitis, and treatment with pentoxifylline appears to be a promising anti-inflammatory therapy. Recent studies have indicated antiTNF $\alpha$ therapy, at least for alcoholic hepatitis. Some complementary and alternative medicinal agents, such as milk thistle and S-adenosylmethionine, may be effective in alcoholic cirrhosis. Treatment of the complications of ALD can improve the quality of life and, in some cases, decrease short-term mortality.
\end{abstract}

Copyright $(2005$ S. Karger AG, Basel

\section{KARGER}

Fax +41613061234

E-Mail karger@karger.ch

www.karger.com
(C) 2005 S. Karger AG, Basel

\section{Overview and Prognosis of Alcoholic Liver Disease}

Alcoholic liver disease (ALD) remains a major health problem worldwide. The prognosis of individual patients with ALD depends on the degree of pathological injury, the patient's nutritional status, the presence of complications of advanced disease, other comorbid conditions such as hepatitis $\mathrm{C}$ virus infection, and the patient's ability to eliminate destructive patterns of drinking. In studies that have examined the natural history of ALD on the basis of histological characteristics at diagnosis, patients with fatty liver have had the best outcome $(70-80 \%$ survival rate at 4-5 years); those with alcoholic hepatitis (AH) or cirrhosis, an intermediate outcome $(50-75 \%$ survival rate at 4-5 years), and those with cirrhosis combined with $\mathrm{AH}$, the worst outcome (30-50\% survival rate at 4-5 years) [1]. Considering all patients with ALD as a single group, the average 1- and 5-year survival rates are approximately 80 and 50\%, respectively [2]. Alcoholic cirrhosis also appears to be an independent risk factor for hepatocellular carcinoma, albeit a weaker one than viral hepatitis [3-6]. Among alcoholics, men older than 50 years of age appear to be most vulnerable to the development of hepatocellular carcinoma [7].

In addition to providing general counseling for patients, estimating the prognosis of patients with ALD is particularly important in two clinical settings: (1) deter- 
mining the need for specific therapy in patients with severe $\mathrm{AH}$, and (2) determining the need for liver transplantation in patients with alcoholic cirrhosis.

\section{Alcoholic Hepatitis}

The prognosis of individual patients with $\mathrm{AH}$ can vary dramatically. Patients with severe disease have an extremely high mortality, approaching that of patients with fulminant hepatic failure. Clinical features associated with severe disease include hepatic encephalopathy, marked prolongation of prothrombin time values, elevation in serum bilirubin above $25 \mathrm{mg} / \mathrm{dl}$, depressed serum albumin, elevated serum creatinine, and older age. Maddrey et al. [8] discovered a simple formula called the discriminant function $(\mathrm{DF})([4.6 \times$ prothrombin time - control value (seconds)] + serum bilirubin $(\mathrm{mg} / \mathrm{dl}))$ that is extremely useful in identifying patients with poor shortterm survival. Three prospective studies have demonstrated that patients with DF values of $>32$ have an extremely poor prognosis, with 1-month mortality rates of $35-45 \%$ [9-11]. In contrast, patients with lower DF values have short-term survival rates of $90-100 \%[8,12]$. Other important prognostic variables in patients with severe $\mathrm{AH}$ are the presence of spontaneous hepatic encephalopathy and hepatorenal syndrome [9, 13, 14]. Onemonth mortality in patients with spontaneous hepatic encephalopathy is approximately $50 \%$ and in those with hepatorenal syndrome is $75 \%[9-11,13,14]$.

\section{Alcoholic Cirrhosis}

The clinical tool most widely used to determine prognosis in patients with alcoholic cirrhosis is the Child-Turcotte-Pugh (CTP) classification. This simple classification system, which was designed to stratify the risk of portacaval shunt surgery in cirrhotics with variceal bleeding, has gained favor over the past decade as a rapid method for determining the prognosis of patients with various chronic liver diseases. The CTP is as effective as quantitative liver function tests and disease-specific prognostic models in determining short-term prognosis in groups of patients awaiting liver transplantation $[15,16]$. Although its limitations have been well described, the CTP has been widely adopted for risk-stratifying patients with cirrhosis because of its simplicity and ease of use. Five-year survival rates of patients with alcoholic cirrhosis vary dramatically by CTP classification at the time of clinical presentation [17].

The development of ascites, variceal bleeding, hepatic encephalopathy, spontaneous bacterial peritonitis (SBP), or hepatorenal syndrome also has a significant impact on the prognosis of patients with alcoholic cirrhosis. The 5year survival of individuals who develop any of these complications is only $20-50 \%$ of that for patients with compensated cirrhosis [18]. The most ominous complications are SBP and rapid-onset hepatorenal syndrome. Less than half of those who develop SBP can be expected to survive 1 year, while the median survival of patients with hepatorenal syndrome is less than 2 weeks [19, 20].

Other models that have been used to predict prognosis in patients with alcoholic cirrhosis are the proportionalhazards model developed by Poynard et al. [16, 17] (Beclere model) and the prognostic model for end-stage liver disease (MELD) developed by investigators at the Mayo Clinic [21]. The Beclere model, which was developed from a database of 818 patients with alcoholic cirrhosis who were followed prospectively for 4 years, includes serum bilirubin, serum albumin, patient age, and hepatic encephalopathy $[16,17]$. The MELD model, which was originally developed to assess short-term prognosis in patients undergoing transjugular intrahepatic portosystemic shunts, includes serum creatinine, the international normalized ratio for prothrombin time, and serum bilirubin values [21]. A modification of this model is now used to prioritize patients for donor allocation in the United States. The MELD score has been shown to be useful in predicting short-term survival in groups of patients with various liver diseases [22].

\section{Therapy for ALD}

Unfortunately, in the United States there is no FDAapproved therapy for either alcoholic cirrhosis or AH. However, several therapies have been employed to either treat the complications of ALD or, in some cases, attempt to treat the liver damage itself. We will discuss therapies available for which there are data from large randomized human studies, as well as selected promising new approaches based on results from basic research studies.

\section{Treatment of Decompensation}

In the absence of hepatocellular carcinoma, death due to ALD is often secondary to the disease per se. As mentioned above, there is a host of effects associated with a failing liver (e.g., portal hypertension, hepatic encephalopathy, ascites, hepatorenal syndrome, and esophageal varices) that are the major causes of clinical complications and mortality in ALD. These complications may be due to liver failure and portal hypertension or to factors 
Fig. 1. Selected clinical complications of

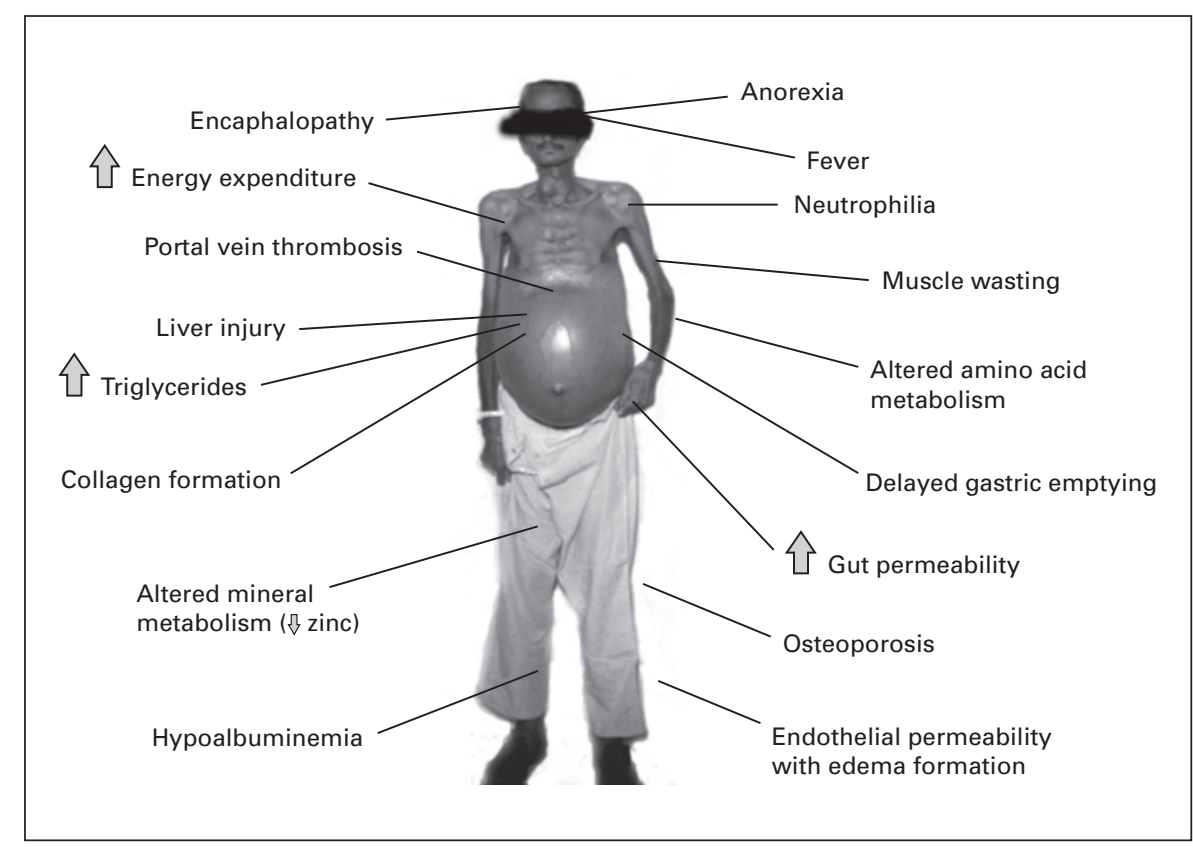
ALD in which TNF may play a role.

such as tumor necrosis factor (TNF) that play an etiologic role in the liver disease (fig. 1). These complications are very common in alcoholic cirrhotics. For example, Lucena et al. [23] showed incidence rates for ascites, hepatic encephalopathy and variceal bleeding of 49, 24 and $22 \%$, respectively, in cirrhotic patients in a multicenter study in Spain. Powell and Klatskin [24] showed that 5year survival in ALD patients with overt hepatic decompensation (drinkers/abstainers) was 34 and 60\%, respectively; in contrast, compensated cirrhotics (drinkers/ abstainers) had a 5-year survival of 68 and $89 \%$, respectively. Therefore, clinical management of these complications of cirrhosis is critical for the long-term survival of the patient [25-28].

\section{Abstinence}

Abstinence from alcohol is vital in order to prevent further ongoing liver injury, fibrosis and possibly hepatocellular carcinoma. Abstinence causes total resolution of alcoholic steatosis. There are limited studies evaluating the effects of abstinence from alcohol on the progression of ALD, and these are retrospective, non-randomized trials. However, virtually all studies show the beneficial effects of abstinence. As mentioned above, early studies from the program of Powell and Klatskin [24] showed that patients with jaundice or ascites especially benefited from abstinence. In a recent small series of patients with alcoholic cirrhosis who were followed for 4 years or until death, Merkel et al. [29] showed that the cumulative probability of survival was $87 \%$ in persistent abstainers and only $55 \%$ in persistent drinkers. Moreover, recent data from recent Veterans Administration (VA) Cooperative Studies suggest that reducing, but not stopping, ethanol consumption also improves projected survival in ALD [30]. Thus, abstinence, or a major reduction in drinking, should be encouraged in all patients with ALD. Individual patients may exhibit major clinical improvement with abstinence (fig. 2). Newer agents to improve abstinence, such as Naltrexone and Acamprosate, have been shown to have efficacy in some chronic alcoholics, however there are no large multicenter studies evaluating these drugs in patients with ALD.

\section{Nutrition Therapy}

Alcoholic Hepatitis

Malnutrition is prevalent in liver disease, especially in the more severe forms of chronic liver disease. The etiology of this malnutrition is multifactorial (table 1). Probably the most extensive studies on the nutritional status of patients with liver disease are large studies by the VA Cooperative Studies Program dealing with patients having $\mathrm{AH}$ [31]. The first of these studies demonstrated that virtually every patient with $\mathrm{AH}$ had some degree of malnutrition [32]. Patients had a mean alcohol consumption of $228 \mathrm{~g}$ /day (with almost 50\% of energy intake coming from alcohol). The severity of liver disease generally cor- 
Fig. 2. Patient with alcoholic cirrhosis before and after 3 years of abstinence.
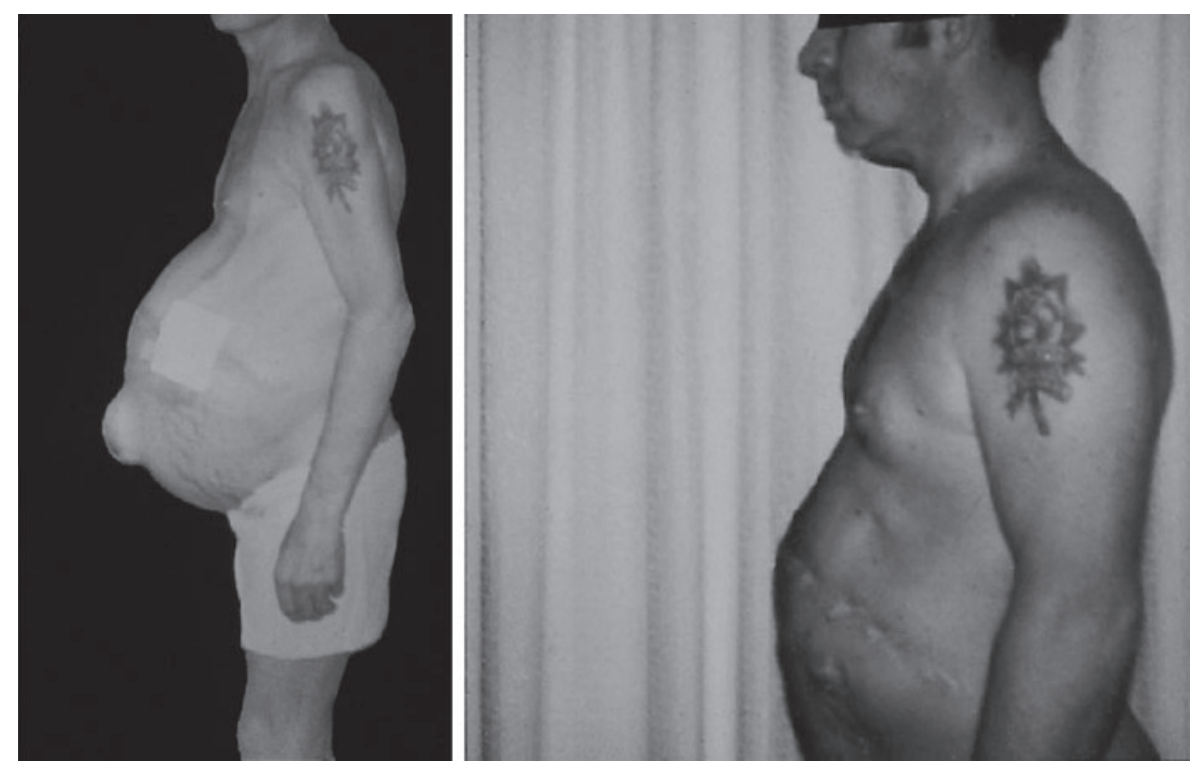

Table 1. Selected causes of malnutrition

\author{
Anorexia \\ Altered taste/smell \\ Nausea/vomiting \\ Diarrhea/malabsorption \\ Poor food availability/quality \\ Metabolic disturbances (e.g., hypermetabolism/catabolism) \\ Cytokine effects \\ Complications of liver disease (PSE, ascites, GI bleeding) \\ Unpalatible diets (Na, protein) \\ Fasting for procedures
}

PSE $=$ Partial splenic embolization; GI = gastrointestinal.

related with the severity of malnutrition. Similar data were generated in a follow-up VA study on AH (No. 275). In both of these studies, patients were given a balanced 2,500-kcal hospital diet, monitored carefully by a dietitian, and were encouraged to consume the diet. In the second study, patients in the therapy arm of the protocol also received an enteral nutritional support product high in branched-chain amino acids as well as the anabolic steroid oxandrolone $(80 \mathrm{mg} /$ day $)$. Patients were not fed by tube if voluntary oral intake was inadequate in either study (probably a study design flaw, in retrospect). Voluntary oral food intake correlated in a stepwise fashion with 6-month mortality data. Thus, patients who volun- tarily consumed $>3,000 \mathrm{kcal} /$ day had virtually no mortality, whereas those consuming $<1,000 \mathrm{kcal} /$ day had a $>80 \%$ 6-month mortality [31]. Moreover, the degree of malnutrition correlated with the development of serious complications such as encephalopathy, ascites and hepatorenal syndrome [31].

\section{Cirrhosis}

Interest in nutrition therapy for cirrhosis was stimulated when Patek et al. [33] and demonstrated that a nutritious diet improved the 5-year outcome of patients with alcoholic cirrhosis compared with patients consuming an inadequate diet. Several recent studies have further supported the concept of improved outcome with nutritional support in patients with cirrhosis. Hirsch et al. [34] demonstrated that outpatients taking an enteral nutritional support product ( $1,000 \mathrm{kcal}, 34 \mathrm{~g}$ protein $)$ had significantly improved protein intake and significantly fewer hospitalizations. These same investigators subsequently gave an enteral supplement to outpatients with alcoholic cirrhosis and observed an improvement in nutritional status and immune function [35]. In the VA Cooperative Study No. 275 on nutritional support in ALD using both an anabolic steroid and an enteral nutritional supplement, improved mortality was seen with the combination of oxandrolone plus supplement in patients who had moderate protein-energy malnutrition [31]. Those with severe malnutrition did not significantly benefit from therapy, possibly because their malnutrition was so 
advanced that no intervention, including nutrition, could help. Studies by Kearns et al. [36] showed that patients with ALD hospitalized for treatment and given an enteral nutritional supplement via tube feeding had significantly improved serum bilirubin levels and liver function as assessed by antipyrine clearance. Moreover, a major randomized multicenter study of enteral nutrition versus steroids in patients with $\mathrm{AH}$ showed similar overall initial outcomes, and less long-term infections in the nutrition group [10]. Thus, traditional nutritional supplementation clearly improves nutritional status and, in some instances, hepatic function and other outcome indicators in $\mathrm{AH} /$ cirrhosis.

\section{Drug Therapies of Likely Benefit}

Corticosteroids

Corticosteroids have been the most extensively studied form of therapy for $\mathrm{AH}$, but their role remains limited [37]. The rationale for steroid use is to decrease the immune response and pro-inflammatory cytokine response. Most randomized studies have supported the use of corticosteroids in moderate/severe $\mathrm{AH}$ [38], but the large multicenter VA study by Mendenhall et al. [31] yielded negative results. Most meta-analyses support the use of steroids for severe acute $\mathrm{AH}$ including the most recent study by Mathurin et al. [12]. This study reported significantly improved survival at 28 days ( 85 vs. $65 \%$ ) in severely ill AH patients having a DF of $>32$. This survival advantage may extend to 1 year, but not 2 . Independent prognostic factors associated with death at 28 days in this meta-analysis were steroid treatment, age, and creatinine. It is important to note that the patients studied were highly selected, and infections (e.g., SBP), gastrointestinal bleeding, and many other common complications were exclusions for entry into these studies. Most investigators agree that if corticosteroids are to be used, they should be reserved for those with relatively severe liver disease (DF >32), and possibly those with hepatic encephalopathy. Steroids have well-documented side effects, including enhancing the risk of infection which is already substantial in patients with $\mathrm{AH}$. Thus, a major disadvantage to corticosteroids is their lack of applicability in many patients with $\mathrm{AH}$.

\section{Pentoxifylline}

Pentoxifylline (PTX) is a nonselective phosphodiesterase inhibitor which increases intracellular concentrations of adenosine $3^{\prime}, 5^{\prime}$-cyclic monophosphate (cAMP) and guanosine $3^{\prime}, 5^{\prime}$-cyclic monophosphate (cGMP), and decreases production of pro-inflammatory chemokines/ cytokines including TNF. Akriviadis et al. [9] from the University of Southern California Liver Unit performed a prospective, randomized, double-blind clinical trial of PTX in severe $\mathrm{AH}(\mathrm{DF}>32)$. Forty-nine patients received $400 \mathrm{mg}$ PTX orally 3 times daily and 52 received placebo (vitamin $\mathrm{B}_{12}$ ) for 4 weeks. PTX treatment improved survival. Twelve PTX patients died (24.5\%) compared with $24(46 \%)$ placebo patients. PTX also decreased hepatorenal syndrome as a cause of death. Six of the $12(50 \%)$ PTX-treated patients who died did so of renal failure compared with 22 of the 24 (92\%) control patients who died of renal failure. Multivariate analysis revealed age, serum creatinine at randomization, and treatment with PTX as independent factors associated with survival. We regularly use PTX in patients with AH and alcoholic cirrhosis because of its anti-inflammatory properties, its protective effects against hepatorenal syndrome, and its excellent safety profile.

\section{Anti-TNF Therapy}

Dysregulated cytokine metabolism was described in $\mathrm{AH}$ long before it was recognized in inflammatory bowel disease and rheumatoid arthritis. However, anti-TNF therapy has been an FDA-approved, highly effective treatment for both of these diseases for several years. An initial concern in ALD arose from early observations that low 'basal' amounts of TNF were important for liver regeneration [39]. Thus, many investigators took the tack that downregulating but not totally blocking TNF activity would be a preferred therapeutic intervention. Indeed, many therapies used in ALD (corticosteroids, PTX, Sadenosylmethionine, etc.) decrease but do not abolish TNF activity.

Because anti-TNF antibody has been shown to block development of alcohol-induced liver injury in rats, it is now being used in small clinical trials in patients with AH. A study from Europe involved 12 patients with moderate to severe $\mathrm{AH}$ who were given Infliximab (anti-TNF antibody) $5 \mathrm{mg} / \mathrm{kg}$ as a single 2-hour infusion. Ten of the 12 patients were alive at a median of 15 months [40]. Pilot data from a small US open-label trial of etanercept, a TNF receptor antagonist, also showed safety in patients with less severe $\mathrm{AH}$, and a multicenter trail studying this agent is being funded by the National Institutes of Health [41]. On the other hand, a large double-blind randomized control trial from France using either prednisolone treatment or prednisolone treatment plus high-dose Infliximab in patients with acute $\mathrm{AH}$ was terminated due to increased infectious complications in the combined therapy group [40]. Thus, etanercept may be more attractive 
than Infliximab in ALD because of its shorter duration of action. However, there remains the theoretic concern of completely blocking TNF activity over a long duration in relation to increased infections and impaired liver regeneration. On a related point, the question of whether or not anti-TNF therapies will be beneficial for cirrhosis as well as $\mathrm{AH}$ remains to be determined. Until more data are available, specific anti-TNF therapy should optimally be performed in the context of a clinical trial [41].

\section{Drug Therapies of Unlikely Benefit}

Colchicine

Colchicine has been suggested as a treatment for ALD because of its anti-fibrotic effects. It has many potential therapeutic mechanisms of action including inhibition of collagen production, enhancement of collagenase activity, and anti-inflammatory functions. Initial positive studies by Kershenobich et al. [42] led to a large VA Cooperative Study evaluating colchicine therapy in alcoholic cirrhosis that has recently been completed. Results showed no beneficial effect on either overall mortality or liver-related mortality [43]. A recent smaller study from Europe also showed no beneficial effects of colchicine [44]. Thus, despite initial enthusiasm and biochemical rationale for use of this drug, it does not appear to be effective in ALD.

\section{Propylthiouracil (PTU)}

Chronic alcohol feeding in experimental animals produces a hypermetabolic state with increased oxygen consumption. This may lead to relative hypoxia, especially in the central lobular area, or zone 3, of the liver. PTU has been postulated to attenuate this hypermetabolic state, to function as an antioxidant, and to improve portal blood flow. This prompted a long-term study of PTU therapy by Orrego et al. [1] in over 300 patients with a variety of types of liver disease, including ALD. In the total study population, mortality was reduced by nearly $50 \%$ in patients receiving PTU [1]. A recent Cochran review evaluated PTU therapy for ALD, including alcoholic steatosis, alcoholic fibrosis, $\mathrm{AH}$ and/or cirrhosis [45]. Combining the results of 6 randomized clinical trials which included 710 patients, no significant effects of PTU versus placebo on mortality or liver-related mortality, complications of liver disease, or liver histology were shown [45].

\section{Dilinoleoylphosphatidylcholine}

Dilinoleoylphosphatidylcholine (a form of lecithin/ soybean extract) has antioxidant, anti-fibrotic, and anticytokine activity in experimental rat models of ALD [46].
However, a recently completed VA Cooperative Study failed to show significant benefit in human ALD [30]. It should be noted that in that study, the partial abstinence observed in all patients, regardless of whether or not they received placebo or drug, may have confounded any possible protective effects (see Abstinence above) [30].

\section{Anabolic Steroids}

ALD is associated with severe muscle wasting. While in part mediated by nutritional deficiencies, decreased functional levels of anabolic and androgenic steroids in alcoholics also occur [47], which may contribute to the loss of muscle mass. Utilizing the Cochrane database [48], the results of 5 randomized clinical trials on 499 patients with $\mathrm{AH}$ and/or cirrhosis demonstrated no significant effects of anabolic-androgenic steroids on mortality, liverrelated mortality, and liver histology. There was also no effect of anabolic-androgenic steroids on a number of other outcome measures. Furthermore, a slight increase in the risk of occurrence of serious adverse events was observed with the use of these hormones. Thus, the lack of any observed benefit coupled with potential iatrogenic effects decreases enthusiasm for the use of these hormones in ALD.

\section{Complementary and Alternative Medicines}

Silymarin (Milk Thistle)

Silymarin is probably the most widely used form of complementary and alternative medicine in the treatment of liver disease in the US. It has antioxidant activities, it protects against lipid peroxidation, and it has antiinflammatory and anti-fibrotic effects. Large controlled trials of silymarin have been performed in Europe, with varying results. Ferenci et al. [49] evaluated 170 patients with cirrhosis in a treatment program (140 mg t.i.d.) with a mean duration of 41 months. They observed a positive beneficial effect, especially in patients with alcoholic cirrhosis and in those with milder disease (Child's A category). On the other hand, a study by Pares et al. [50] found no beneficial effects ( $150 \mathrm{mg}$ silymarin t.i.d.) in a study of 200 patients with alcoholic cirrhosis, some of whom also had hepatitis C. Both of these trials had major shortcomings, including high dropout rates and compliance issues. In all studies performed thus far, the drug appears quite safe.

\section{S-Adenosylmethionine (SAMe)}

$\mathrm{SAMe}$, or AdoMet, is an obligatory intermediate in the conversion of methionine to cysteine in the hepatic transsulfuration pathway. SAMe is a precursor for syntheses 
of polyamines, choline, and glutathione (GSH), and it is the major methylating agent for a vast number of molecules via specific methyltransferases [51]. Patients with ALD have elevated plasma methionine levels, markedly delayed clearance of an oral methionine load, and decreased hepatic methionine adenosyltransferase (MAT) activity (the enzyme responsible for conversion of methionine to SAMe). Hepatic-specific MAT is highly sensitive to oxidative stress, and it is likely that subnormal hepatic MAT activity reported in ALD is due to oxidation of the active site [52]. Studies from our laboratories have shown that SAMe downregulates production of the cytotoxic pro-inflammatory cytokine, TNF, in animal models of liver injury and in peripheral blood monocytes or macrophage cell lines in vitro [53, 54]. Mato et al. [55] reported that patients with alcoholic liver cirrhosis who were randomized to receive $\mathrm{SAMe}(1,200 \mathrm{mg} /$ day orally $)$ for 2 years had decreased liver mortality/liver transplantation (16 vs. $30 \%)$ compared to the placebo-treated group.

\section{Vitamin E}

Vitamin E deficiency has been well documented in ALD [56]. Vitamin E has been used extensively with hepatoprotective effects in experimental models of liver injury such as that induced by carbon tetrachloride or ischemia. Vitamin $\mathrm{E}$ has multiple potential beneficial effects including membrane stabilization [56], reduced NFKB activation and TNF production [57], and inhibition of hepatic stellate cell activation and collagen production [58]. Unfortunately, a major randomized study of vitamin E in ALD showed no benefit, but may have used an inadequate dose $(500 \mathrm{mg})$ [59]. A smaller study with a higher dose $(1,000 \mathrm{mg})$ of vitamin $\mathrm{E}$ in AH observed some normalization of serum hyaluronic acid levels, but without significant changes in indices of liver damage [60]. Furthermore, recent meta-analyses suggest that high-dose vitamin $\mathrm{E}$ supplementation is associated with an increase in overall mortality $[61,62]$, raising concerns of prolonged usage of this drug in humans. It should be noted that most clinical trials with vitamin $\mathrm{E}$ have been performed in the absence of concomitant citamin C (ascorbic acid) supplementation, which is required for catalytic maintenance of the antioxidant function of vitamin $\mathrm{E}$.

\section{GSH Prodrugs}

GSH is a tripeptide which is synthesized from glutamate, cysteine, and glycine. GSH prodrugs have been used extensively in virtually every known experimental model of hepatotoxicity with beneficial results [63]. The

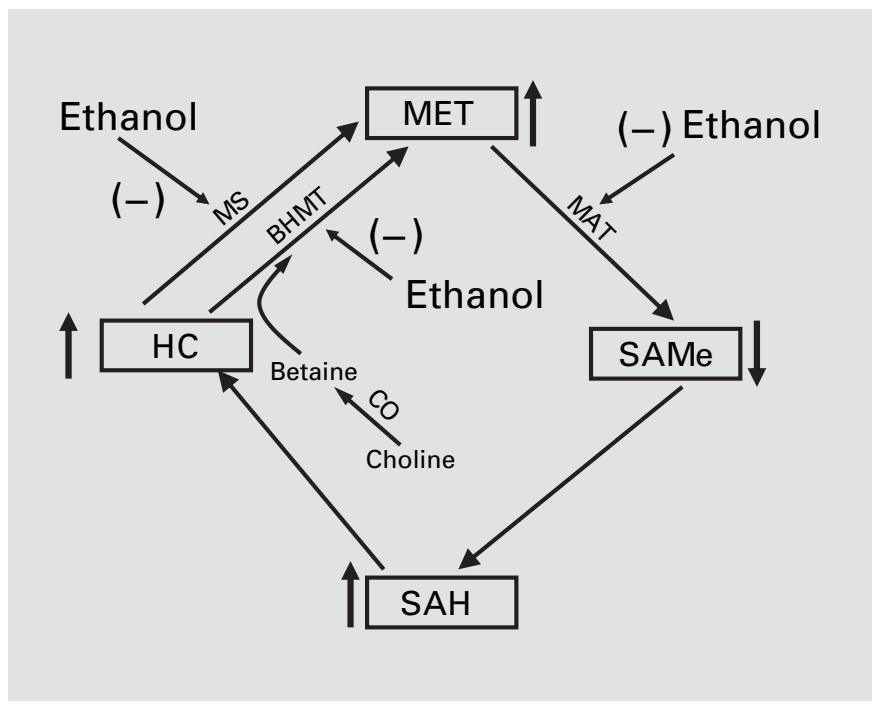

Fig. 3. Effect of ethanol on methionine metabolism.

GSH prodrug, $\mathrm{N}$-acetylcysteine (given as Mucomyst), is the standard therapy for acetaminophen toxicity in humans. Maintaining adequate hepatocyte GSH levels has been documented to prevent acetaminophen liver injury. GSH prodrugs can directly affect the hepatocyte, and they can also positively modulate pro-inflammatory cytokine production with inhibition of cytokines such as TNF and IL-8 [57]. Pena et al. [64] demonstrated that the GSH prodrug, procysteine, can increase whole blood GSH and inhibit monocyte TNF and IL-8 production when given intravenously to stable alcoholic cirrhotics. Similarly, procysteine was shown to protect against alcohol-induced liver injury in the intragastric ethanol-feeding model [65]. However, large, randomized studies of GSH prodrugs using mortality as an outcome indicator are lacking in ALD.

\section{Other Selected Potential Therapies}

As mentioned above, alcohol alters methionine metabolism in the liver. In addition to increasing methionine and decreasing SAMe levels, there is an accumulation of homocysteine after alcohol exposure (fig. 3) [66, 67]. In human alcoholics, the mRNA levels of the 2 enzymes responsible for remethylation of homocysteine, methylfolate-homocysteine methyltransferase (methionine synthase) and betaine-homocysteine methyltransferase are decreased [68]. Furthermore, levels of precursors required for remethylation of homocysteine (e.g. fo- 
late and vitamin $\mathrm{B}_{12}$ ) are also decreased in alcoholics [67]. For this reason, it has been proposed that supplementation with either folic acid [69] or betaine may also be protective in ALD. Indeed, experimental liver damage in both rats and mice is protected by betaine supplementation [70]. While much of the hypothesized protective effect of betaine has focused on decreasing homocysteine levels, recent work by Song et al. [71] indicates that Sadenosyl homocysteine may contribute the sensitization of hepatocytes to $\mathrm{TNF} \alpha$-induced cell killing.

Another potential dietary supplement is zinc. It is well known that circulating levels of zinc are depleted in patients with $\mathrm{AH}$ and cirrhosis $[72,73]$. The zinc depletion caused by alcohol consumption appears to be mediated both at the level of absorption and excretion [74, 75]. Further, in animal models of alcohol-induced liver damage, zinc supplementation prevented liver damage, oxidative stress and inflammatory cytokine (TNF $\alpha$ ) production caused by alcohol feeding [76]. Zinc also prevented gastrointestinal tract damage caused by repeated acute doses of alcohol in mice [77]. There are limited clinical data supporting the hypothesis that zinc supplementation is potentially therapeutic against some effects of alcoholic cirrhosis [76], but larger studies are needed to validate this point.

\section{The Role of Liver Transplantation}

There have been multiple recent studies and reviews concerning liver transplantation in patients with severe alcoholic cirrhosis [78-82]. There is a well-documented organ shortage for liver transplantation, and there are serious ethical issues concerning this controversial area that have precipitated these studies. Hepatitis C and ALD are the two major reasons for liver transplantation in the US. Data clearly demonstrate that patients transplanted for ALD do (short- and long-term) as well as patients transplanted for hepatitis $\mathrm{C}$ or other types of liver disease. However, AH clearly is not an indication for liver transplantation at the current time. Virtually all centers require that alcoholic patients undergo formal psychiatric evaluation and treatment prior to transplantation. Many centers impose a ' 6 -month rule' of abstinence before being considered for orthotopic liver transplantation, however most centers also show some flexibility with this rule. It is unusual for ALD alone to be the cause of graft failure. The majority of patients with ALD are not listed for liver transplantation for multiple reasons including continued alcohol consumption, improvement in liver function with abstinence, lack of interest, etc. Patients with ALD appear to have a higher incidence of certain malignancies of the upper airway and upper digestive tract. Therefore, these patients should be screened for these processes prior to transplantation and monitored carefully after transplantation. Data suggest that, following transplantation, patients who had ALD and those who were transplanted for other reasons consume alcohol at relatively similar rates, although those who had ALD may consume greater amounts. The rate of alcohol use increases over time for all transplant recipients. Some centers use multi-stage screening processes with risk stratification to select patients with low rates of recidivism. Clearly, more studies are required to refine our predictive capabilities for both recidivism and noncompliance. Quality of life appears to improve after liver transplantation due to any etiology, although those with non-alcohol etiologies may improve more.

\section{Conclusions}

The optimal management of all patients with ALD begins with a dramatic reduction or elimination of alcohol intake, which often can be successfully accomplished using 'brief interventions' by a nurse, primary care physician, or gastroenterologist. Abstinence can have a profound impact on survival even in patients with decompensated cirrhosis. Other lifestyle modifications should be employed (e.g., weight loss, smoking cessation) where appropriate. Although palliative, successful treatment of decompensation has significant short-term survival benefits. Proper nutrition and nutrition support have consistently been shown to be important. Certain drugs (e.g., corticosteroids or PTX) may be effective in selected patients. While no FDA-approved therapies are available, many new agents, such as anti-TNF antibody, are under investigation. Lastly, there are a host of complimentary and alternative medicine approaches that may prove beneficial in treating the underlying disease. Transplantation is life-saving in certain abstinent patients with end-stage liver disease.

\section{Acknowledgment}

Research support from the National Institute of Alcohol Abuse and Alcoholism (NIAAA) is gratefully acknowledged. 


\section{References}

1 Orrego H, Blake JE, Blendis LM, Medline A: Prognosis of alcoholic cirrhosis in the presence and absence of alcoholic hepatitis. Gastroenterology 1987;92:208-214.

2 Mendenhall CL: Alcoholic hepatitis. Clin Gastroenterol 1981;10:417-441.

-3 Schoniger-Hekele M, Muller C, Kutilek M, Oesterreicher C, Ferenci P, Gangl A: Hepatocellular carcinoma in Austria: aetiological and clinical characteristics at presentation. Eur J Gastroenterol Hepatol 2000;12:941-948.

-4 Hassan MM, Hwang LY, Hatten CJ, Swaim M, Li D, Abbruzzese JL, et al: Risk factors for hepatocellular carcinoma: synergism of alcohol with viral hepatitis and diabetes mellitus. Hepatology 2002;36:1206-1213.

-5 Yamauchi M, Nakahara M, Maezawa Y, Satoh S, Nishikawa F, Ohata M, et al: Prevalence of hepatocellular carcinoma in patients with alcoholic cirrhosis and prior exposure to hepatitis C. Am J Gastroenterol 1993;88:39-43.

-6 Neuberger J, Schulz KH, Day C, Fleig W, Berlakovich GA, Berenguer M, et al: Transplantation for alcoholic liver disease. J Hepatol 2002; 36:130-137.

-7 Poynard T, Aubert A, Lazizi Y, Bedossa P, Hamelin B, Terris B, et al: Independent risk factors for hepatocellular carcinoma in French drinkers. Hepatology 1991;13:896-901.

8 Maddrey WC, Boitnott JK, Bedine MS, Weber FL Jr, Mezey E, White RI Jr: Corticosteroid therapy of alcoholic hepatitis. Gastroenterology 1978;75:193-199.

-9 Akriviadis E, Botla R, Briggs W, Han S, Reynolds T, Shakil O: Pentoxifylline improves short-term survival in severe acute alcoholic hepatitis: a double-blind, placebo-controlled trial. Gastroenterology 2000;119:1637-1648.

10 Carithers RL Jr, Herlong HF, Diehl AM, Shaw EW, Combes B, Fallon HJ, et al: Methylprednisolone therapy in patients with severe alcoholic hepatitis: a randomized multicenter trial. Ann Intern Med 1989;110:685-690.

11 Ramond M-J, Poynard T, Rueff B, Mathurin $\mathrm{P}$, Theodore $\mathrm{C}$, Chaput J-C, et al: A randomized trial of prednisolone in patients with severe alcoholic hepatitis. N Engl J Med 1992; 326:507-512.

12 Mathurin P, Mendenhall CL, Carithers RL, Ramond MJ, Maddrey WC, Garstide P, et al: Corticosteroids improve short-term survival in patients with severe alcoholic hepatitis (AH): individual data analysis of the last three randomized placebo controlled double blind trials of corticosteroids in severe AH. J Hepatol 2002;36:480-487.

$\checkmark 13$ Helman RA, Temko MH, Nye SW, Fallon HJ: Alcoholic hepatitis: natural history and evaluation of prednisolone therapy. Ann Intern Med 1971;74:311-321.

$\checkmark 14$ Depew W, Boyer T, Omata M, Redeker A, Reynolds T: Double-blind controlled trial of prednisolone therapy in patients with severe acute alcoholic hepatitis and spontaneous encephalopathy. Gastroenterology 1980;78:524529.
15 Oellerich M, Burdelski M, Lautz H-U, Binder L, Pichlmayr R: Predictors of one-year pretransplant survival in patients with cirrhosis. Hepatology 1991;14:1029-1034.

16 Poynard T, Barthelemy P, Fratte S, Boudjema $\mathrm{K}$, Doffoel M, Vanlemmens C, et al: Evaluation of efficacy of liver transplantation in alcoholic cirrhosis by a case-control study and simulated controls. Lancet 1994;344:502-507.

17 Poynard T, Naveau S, Doffoel M, Boudjema $\mathrm{K}$, Vanlemmens C, Mantion G, et al: Evaluation of efficacy of liver transplantation in alcoholic cirrhosis using matched and simulated controls: 5-year survival. Multi-centre group. J Hepatol 1999;30:1130-1137.

18 Gines P, Quintero E, Arroyo V, Teres J, Bruguera M, Rimola A, et al: Compensated cirrhosis: natural history and prognosis factors. Hepatology 1987; 7:122-128.

19 Andreu M, Sola R, Sitges SA, Alia C, Gallen M, Vila MC, et al: Risk factors for spontaneous bacterial peritonitis in cirrhotic patients with ascites. Gastroenterology 1993;104:11331138.

20 Gines A, Escorsell A, Gines P, Salo J, Jimenez $\mathrm{W}$, Inglada L, et al: Incidence, predictive factors, and prognosis of the hepatorenal syndrome in cirrhosis with ascites. Gastroenterology 1993;105:229-236.

21 Malinchoc M, Kamath PS, Gordon FD, Peine CJ, Rank J, ter Borg PC: A model to predict poor survival in patients undergoing transjugular intrahepatic portosystemic shunts. Hepatology 2000;31:864-871.

22 Kamath PS, Wiesner RH, Malinchoc M, Kremers W, Therneau TM, Kosberg CL, et al: A model to predict survival in patients with endstage liver disease. Hepatology 2001;33:464470.

23 Lucena MI, Andrade RJ, Tognoni G, Hidalgo R, De La Cuesta FS, Spanish Collaborative Study Group on Therapeutic Management In Liver Disease: Multicenter hospital study on prescribing patterns for prophylaxis and treatment of complications of cirrhosis. Eur J Clin Pharmacol 2002;58:435-440.

24 Powell WJ, Klatskin G: Duration and survival in patients with Laennec's cirrhosis. Influence of alcohol withdrawal, and possible effects of recent changes in general management of the disease. Am J Med 1968;44:406-420.

25 Garcia-Tsao G: Current management of the complications of cirrhosis and portal hypertension: variceal hemorrhage, ascites, and spontaneous bacterial peritonitis. Gastroenterology 2001;120:726-748.

26 Riordan SM, Williams R: Treatment of hepatic encephalopathy. N Engl J Med 1997;337: 473-479.

27 Nietsch HH: Management of portal hypertension. J Clin Gastroenterol 2005;39:232-236.

28 Boyer TD: Transjugular intrahepatic portosystemic shunt: current status. Gastroenterology 2003;124:1700-1710.
29 Merkel C, Marchesini G, Fabbri A, Bianco S, Bianchi G, Enzo E, et al: The course of galactose elimination capacity in patients with alcoholic cirrhosis: possible use as a surrogate marker for death. Hepatology 1996;24:820823.

30 Lieber CS, Weiss DG, Groszmann R, Paronetto F, Schenker S: I. Veterans Affairs Cooperative Study of polyenylphosphatidylcholine in alcoholic liver disease: effects on drinking behavior by nurse/physician teams. Alcohol Clin Exp Res 2003;27:1757-1764.

31 Mendenhall CL, Roselle GA, Gartside P, Moritz T: Relationship of protein calorie malnutrition to alcoholic liver disease: a reexamination of data from two Veterans Administration Cooperative Studies. Alcohol Clin Exp Res 1995; 19:635-641.

32 Mendenhall CL, Anderson S, Weesner RE, Goldberg SJ, Crolic KA: Protein-calorie malnutrition associated with alcoholic hepatitis. Veterans Administration Cooperative Study Group on Alcoholic Hepatitis. Am J Med 1984;76:211-222.

33 Patek AJ, Post J, Ralnoff OD: Dietary treatment of cirrhosis of the liver. JAMA 1948;139: 543-549.

- 34 Hirsch S, Bunout D, de la Maza P, Iturriaga H, Petermann M, Icazar G, et al: Controlled trial on nutrition supplementation in outpatients with symptomatic alcoholic cirrhosis. JPEN J Parenter Enteral Nutr 1993;17:119-124.

- 35 Hirsch S, de la Maza MP, Gattas V, Barrera G, Petermann M, Gotteland M, et al: Nutritional support in alcoholic cirrhotic patients improves host defenses. J Am Coll Nutr 1999;18: 434-441.

36 Kearns PJ, Young H, Garcia G: Accelerated improvement of alcoholic liver disease with enteral nutrition. Gastroenterology 1992;102: 200-205.

37 Mathurin P, Duchatelle V, Ramond MJ, Degott C, Bedossa P, Erlinger S, et al: Survival and prognostic factors in patients with severe alcoholic hepatitis treated with prednisone. Gastroenterology 1996;110:1847-1853.

- 38 Cabre E, Rodriguez-Iglesias P, Caballeria J, Quer JC, Sanchez-Pescador R, Pares A, et al: Short- and long-term outcome of severe alcohol-induced hepatitis treated with steroids or enteral nutrition: a multicenter randomized trial. Hepatology 2000;32:36-42.

39 Akerman PA, Cote PM, Yang SQ, McClain C, Nelson S, Bagby G, et al: Long-term ethanol consumption alters the hepatic response to the regenerative effects of tumor necrosis factoralpha. Hepatology 1993;17:1066-1073.

-40 Tilg H, Jalan R, Kaser A, Davies NA, Offner FA, Hodges SJ, et al: Anti-tumor necrosis factor-alpha monoclonal antibody therapy in severe alcoholic hepatitis. J Hepatol 2003;38: 419-425.

41 Menon KV, Stadheim L, Kamath PS, Wiesner $\mathrm{RH}$, Gores GJ, Peine CJ, et al: A pilot study of the safety and tolerability of etanercept in patients with alcoholic hepatitis. Am J Gastroenterol 2004;99:255-260. 
42 Kershenobich D, Vargas F, Garcia-Tsao G, Tamayo RP, Gent M, Rojkind M: Colchicine in the treatment of cirrhosis of the liver. N Engl J Med 1988;318:1709-1713.

-43 Morgan TR, Nemchausky N, Schiff E, Anand BS, Bloor J, Kidao J, et al: Colchicine does not prolong life in patients with advanced alcoholic cirrhosis: results of a prospective, randomized, placebo-controlled, multicenter VA trial (abstract). Gastroenterology 2002;122:641.

- 44 Cortez-Pinto H, Alexandrino P, Camilo ME, Gouveia-Oliveira A, Santos PM, Alves MM, et al: Lack of effect of colchicine in alcoholic cirrhosis: final results of a double blind randomized trial. Eur J Gastroenterol Hepatol 2002; 14:377-381.

-45 Rambaldi A, Iaquinto G, Gluud C: Anabolicandrogenic steroids for alcoholic liver disease: a Cochrane review. Am J Gastroenterol 2002; 97:1674-1681.

-46 Cao Q, Mak KM, Lieber CS: Dilinoleoylphosphatidylcholine decreases acetaldehyde-induced TNF-alpha generation in Kupffer cells of ethanol-fed rats. Biochem Biophys Res Commun 2002;299:459-464.

47 Gluud C: Testosterone and alcoholic cirrhosis. Epidemiologic, pathophysiologic and therapeutic studies in men. Dan Med Bull 1988;35: 564-75.

48 Rambaldi A, Iaquinto G, Gluud C: Anabolic-androgenic steroids for alcoholic liver disease. Cochrane Database Syst Rev 2003; 1:CD003045.

-49 Ferenci P, Dragosics B, Dittrich H, Frank H, Benda L, Lochs H, et al: Randomized controlled trial of silymarin treatment in patients with cirrhosis of the liver. J Hepatol 1989;9: 105-113.

50 Pares A, Planas R, Torres M, Caballeria J, Viver JM, Acero D, et al: Effects of silymarin in alcoholic patients with cirrhosis of the liver: results of a controlled, double-blind, randomized and multicenter trial. J Hepatol 1998;28: 615-621.

-51 Lee TD, Sadda MR, Mendler MH, Bottiglieri T, Kanel G, Mato JM, et al: Abnormal hepatic methionine and glutathione metabolism in patients with alcoholic hepatitis. Alcohol Clin Exp Res 2004;28:173-181.

-52 Sanchez-Gongora E, Ruiz F, Mingorance J, An W, Corrales FJ, Mato JM: Interaction of liver methionine adenosyltransferase with hydroxyl radical. FASEB J 1997;11:1013-1019.

-53 Song Z, McClain CJ, Chen T: S-Adenosylmethionine protects against acetaminophen-induced hepatotoxicity in mice. Pharmacology 2004;71:199-208.

-54 McClain CJ, Hill DB, Song Z, Chawla R, Watson WH, Chen T, et al: S-Adenosylmethionine, cytokines, and alcoholic liver disease. Alcohol 2002;27:185-192.

- 55 Mato JM, Camara J, Fernandez DP, Caballeria L, Coll S, Caballero A, et al: S-adenosylmethionine in alcoholic liver cirrhosis: a randomized, placebo-controlled, double-blind, multicenter clinical trial. J Hepatol 1999;30: 1081-1089.
56 McClain CJ, Hill D, Kugelmas M, Marsano L: Nutrition and liver disease; in Rm B (ed): Present Knowledge in Nutrition. Washington, International Life Sciences Institute, 2001, pp 483-496.

57 Hill DB, Devalaraja R, Joshi-Barve S, Barve S, McClain CJ: Antioxidants attenuate nuclear factor-kappa B activation and tumor necrosis factor-alpha production in alcoholic hepatitis patient monocytes and rat Kupffer cells, in vitro. Clin Biochem 1999;32:563-570.

58 Lee KS, Buck M, Houglum K, Chojkier M: Activation of hepatic stellate cells by TGF alpha and collagen type I is mediated by oxidative stress through c-myb expression. J Clin Invest 1995;96:2461-2468.

59 de la Maza MP, Petermann M, Bunout D, Hirsch S: Effects of long-term vitamin E supplementation in alcoholic cirrhotics. J Am Coll Nutr 1995;14:192-196.

60 Mezey E, Potter JJ, Rennie-Tankersley L, Caballeria J, Pares A: A randomized placebo controlled trial of vitamin $\mathrm{E}$ for alcoholic hepatitis. J Hepatol 2004;40:40-46.

-61 Bjelakovic G, Nikolova D, Simonetti RG, Gluud C: Antioxidant supplements for prevention of gastrointestinal cancers: a systematic review and meta-analysis. Lancet 2004;364: 1219-1228.

62 Miller ER 3rd, Pastor-Barriuso R, Dalal D, Riemersma RA, Appel LJ, Guallar E: Metaanalysis: high-dosage vitamin E supplementation may increase all-cause mortality. Ann Intern Med 2005; 142:37-46.

63 Meister A: Glutathione metabolism and its selective modification. J Biol Chem 1988;263: 17205-17208.

-64 Pena LR, Hill DB, McClain CJ: Treatment with glutathione precursor decreases cytokine activity. JPEN J Parenter Enteral Nutr 1999; 23:1-6.

65 Iimuro Y, Gallucci RM, Luster MI, Kono H, Thurman RG: Antibodies to tumor necrosis factor alfa attenuate hepatic necrosis and inflammation caused by chronic exposure to ethanol in the rat. Hepatology 1997;26:15301537.

66 Hultberg B, Berglund M, Andersson A, Frank A: Elevated plasma homocysteine in alcoholics. Alcohol Clin Exp Res 1993;17:687-689.

67 Cravo ML, Camilo ME: Hyperhomocysteinemia in chronic alcoholism: relations to folic acid and vitamins $\mathrm{B}(6)$ and $\mathrm{B}(12)$ status. Nutrition 2000;16:296-302.

68 Avila MA, Berasain C, Torres L, Martin-Duce A, Corrales FJ, Yang H, Prieto J, Lu SC, Caballeria J, Rodes J, Mato JM: Reduced mRNA abundance of the main enzymes involved in methionine metabolism in human liver cirrhosis and hepatocellular carcinoma. J Hepatol 2000;33:907-914.
69 Halsted CH, Villanueva JA, Devlin AM, Chandler CJ: Metabolic interactions of alcohol and folate. J Nutr 2002;132(suppl):2367S-2372S.

$70 \mathrm{Ji}$ C, Kaplowitz N: Betaine decreases hyperhomocysteinemia, endoplasmic reticulum stress, and liver injury in alcohol-fed mice. Gastroenterology 2003; 124:1488-1499.

71 Song Z, Zhou Z, Uriarte S, Wang L, Kang J, Chen T, et al: S-Adenosylhomocysteine sensitizes to TNF-alpha hepatotoxicity in mice and liver cells: a possible etiological factor in alcoholic liver disease. Hepatology 2004;40:989997.

72 Bode JC, Hanisch P, Henning H, Koenig W, Richter FW, Bode C: Hepatic zinc content in patients with various stages of alcoholic liver disease and in patients with chronic active and chronic persistent hepatitis. Hepatology 1988; 8:1605-1609.

73 McClain CJ, Antonow DR, Cohen DA, Shedlofsky SI: Zinc metabolism in alcoholic liver disease. Alcohol Clin Exp Res 1986;10:582589.

-74 Dinsmore W, Callender ME, McMaster D, Todd SJ, Love AH: Zinc absorption in alcoholics using zinc-65. Digestion 1985;32:238242.

75 Valberg LS, Flanagan PR, Ghent CN, Chamberlain MJ: Zinc absorption and leukocyte zinc in alcoholic and nonalcoholic cirrhosis. Dig Dis Sci 1985;30:329-333.

76 Weismann K, Christensen E, Dreyer V: Zinc supplementation in alcoholic cirrhosis. A double-blind clinical trial. Acta Med Scand 1979; 205:361-366.

77 Lambert JC, Zhou Z, Wang L, Song Z, McClain CJ, Kang YJ: Preservation of intestinal structural integrity by zinc is independent of metallothionein in alcohol-intoxicated mice. Am J Pathol 2004;164:1959-1966.

- 78 Roberts MS, Angus DC, Bryce CL, Valenta Z, Weissfeld L: Survival after liver transplantation in the United States: a disease-specific analysis of the UNOS database. Liver Transpl 2004; 10:886-897.

79 Farges O, Saliba F, Farhamant H, Samuel D, Bismuth A, Reynes M, et al: Incidence of rejection and infection after liver transplantation as a function of the primary disease: possible influence of alcohol and polyclonal immunoglobulin. Hepatology 1996;23:240-248.

80 Pageaux GP, Bismuth M, Perney P, Costes V, Jaber S, Possoz P, et al: Alcohol relapse after liver transplantation for alcoholic liver disease: does it matter? J Hepatol 2003;38:629-634.

81 Buis CI, Wiesner RH, Krom RA, Kremers WK, Wijdicks EF: Acute confusional state following liver transplantation for alcoholic liver disease. Neurology 2002;59:601-605.

82 Bellamy CO, DiMartini AM, Ruppert K, Jain A, Dodson F, Torbenson M, et al: Liver transplantation for alcoholic cirrhosis: long term follow-up and impact of disease recurrence. Transplantation 2001;72:619-626. 